\title{
Determining the extent of the dural sac for the performance of caudal epidural blocks in newborns
}

Research report

Albert-Neels van Schoor ${ }^{1}$, Marius C. Bosman ${ }^{1}$, Gerda Venter ${ }^{1}$, Adrian T. Bösenberg ${ }^{2}$

1 Department of Anatomy, School of Medicine, Faculty of Health Sciences, University of Pretoria, Pretoria, South Africa.

${ }^{2}$ Department Anesthesiology and Pain Management, Seattle Children's Hospital, Seattle, USA.

\section{Correspondence to:}

Prof. A.N. van Schoor,

Department of Anatomy, School of Medicine, Faculty of Health Sciences, University of Pretoria, 9 Bophelo Road, Pretoria 0001, South Africa. albert.vanschoor@up.ac.za

What is already known:

- It is important to have a clear understanding of the level at which the dural sac ends and the distance from the sacral hiatus before attempting to perform a caudal block, or inserting a continuous caudal epidural catheter in a neonate or infant.

- With differential growth of the vertebral column and spinal cord, it is thought that the dural sac migrates from its S3/S4 level in a newborn to the S1/S2 level by 1 year of age.

What this article adds:

- The distance between the sacral hiatus and the dural sac is short in newborns

- The risk of inadvertent dural puncture in neonates, particularly if sharp intravenous needles are used, increases when the needle is advanced more than $5 \mathrm{~mm}$. 


\section{Abstract}

Background: Information regarding the position and relationship of vital structures within the caudal canal is important for anesthesiologists who perform a caudal block. This information can be acquired by anatomical dissection, with ultrasound technology, or radiological studies (CT scan, MRI).

Aim: The aim of this study was to determine the position of the dural sac in neonates by measuring the distance of the termination of the dural sac from the apex of the sacral hiatus in neonatal cadavers.

Methods: After careful dissection, the distance from the apex of the sacral hiatus to the dural sac was measured in a sample of neonatal cadavers.

Results: In 39 neonatal cadavers, the mean distance from the apex of the sacral hiatus to the dural sac was $10.45 \mathrm{~mm}$. The range of this distance was between $4.94 \mathrm{~mm}$ and $26.28 \mathrm{~mm}$. The mean distance for females was $9.64 \mathrm{~mm}$ (range from 6.66 to 15.09); that for males was $10.90 \mathrm{~mm}$ (range between 4.94 and 26.28). Linear regression with the log of this distance as the outcome variable gave an estimated $3.3 \%$ increase in the distance for each $1 \mathrm{~cm}$ increase in the length of the neonate $(95 \%$ $\mathrm{Cl}$ for this proportion was 1.91 to 4.71 ).

Conclusion: Anesthesiologists should be aware of the short distance between the sacral hiatus and the dural sac when performing caudal blocks, the shortest distance was $4.94 \mathrm{~mm}$. Armed with this knowledge caudal techniques should be modified to improve the safety and reduce the risk of complications, such as dural puncture.

Key words: anatomy; caudal epidural anesthesia; caudal canal; sacral canal; dura mater; dural sac 


\section{Introduction}

Caudal block remains the most popular regional anesthesia technique in infants and children. Although relatively uncommon, unintended dural puncture can occur when performing a single-shot caudal block or when threading a caudal catheter for a continuous infusion within the caudal part of the vertebral canal - also known as the caudal canal (1-3). The reported incidence is low approximately 1:1000 - depending on the age group reported (4-8).

Most anesthesiologists introduce a caudal needle or an intravenous cannula into the sacral hiatus at an angle; or change the angle and advance the needle once the sacrococcygeal membrane has been penetrated. These maneuvers potentially increase the risk of dural or vascular puncture.

The level at which the dural sac ends and the distance from the sacral hiatus is useful information to have before attempting to perform a caudal block, or inserting a continuous caudal epidural catheter in a neonate or infant. Various methods have been used to determine the level of the dural sac. These include ultrasound imaging, magnetic resonance imaging (MRI), CT scan or anatomical dissections.

With differential growth of the vertebral column and spinal cord, it is thought that the dural sac migrates from its $S 3 / S 4$ level in a newborn to the $S 1 / S 2$ level of the adult by 1 year of age (9). Adewale and co-workers studied the caudal (sacral extradural) space in 41 children, weighing between $8 \mathrm{~kg}-80 \mathrm{~kg}$, ages 10 months to 18 years, using MRI scans. They found that the distance from the apex of the sacral hiatus to the dural sac was shorter in males, measuring $27.9 \mathrm{~mm} \pm 8.0 \mathrm{~mm}$ (mean \pm standard deviation) than in females, where the average distance was $33.2 \mathrm{~mm} \pm 11.5 \mathrm{~mm}(3)$.

Information regarding its distance in neonates is lacking. The distance that a needle can be safely inserted while performing a caudal block on neonates or young infants would be useful information for anesthesiologists in clinical practice. The primary aim of this study was therefore to determine the position of the dural sac in neonates by measuring the distance of the termination of the dural sac from the apex of the sacral hiatus in a sample of formalin-fixed neonatal cadavers. 


\section{Materials \& methods}

A sample of 39 neonatal cadavers, which included 25 males and 14 females, was used. The mean length was $0.42 \mathrm{~m} \pm 0.07 \mathrm{~m}$ (mean \pm standard deviation), mean weight was $1.61 \mathrm{~kg} \pm 0.86 \mathrm{~kg}$, and ranged between $1 \mathrm{~kg}$ and $4 \mathrm{~kg}$ (minimum and maximum values).

All cadavers were obtained according to the South African National Health Act, number 61 of 2003 and stored in the Department of Anatomy, University of Pretoria, South Africa for research and teaching purposes. Ethical clearance to perform this study (dissection of neonatal cadavers) was obtained from the Research Ethics Committee of the Faculty of Health Sciences, University of Pretoria.

The lumbar and sacral regions of the cadavers were carefully dissected to expose the laminae and spinous processes of the lumbar vertebrae and sacrum. Prior to dissecting the laminae, a pin was placed at the apex of the sacral hiatus and care was taken not to disturb this pin during the dissection.

Using a scalpel, the laminae of the sacrum and the lumbar vertebrae were cut and the spinous processes were carefully removed, effectively exposing the dural sac within the vertebral canal (see Figure 1). A second pin was then placed at the point where the dural sac ended. 


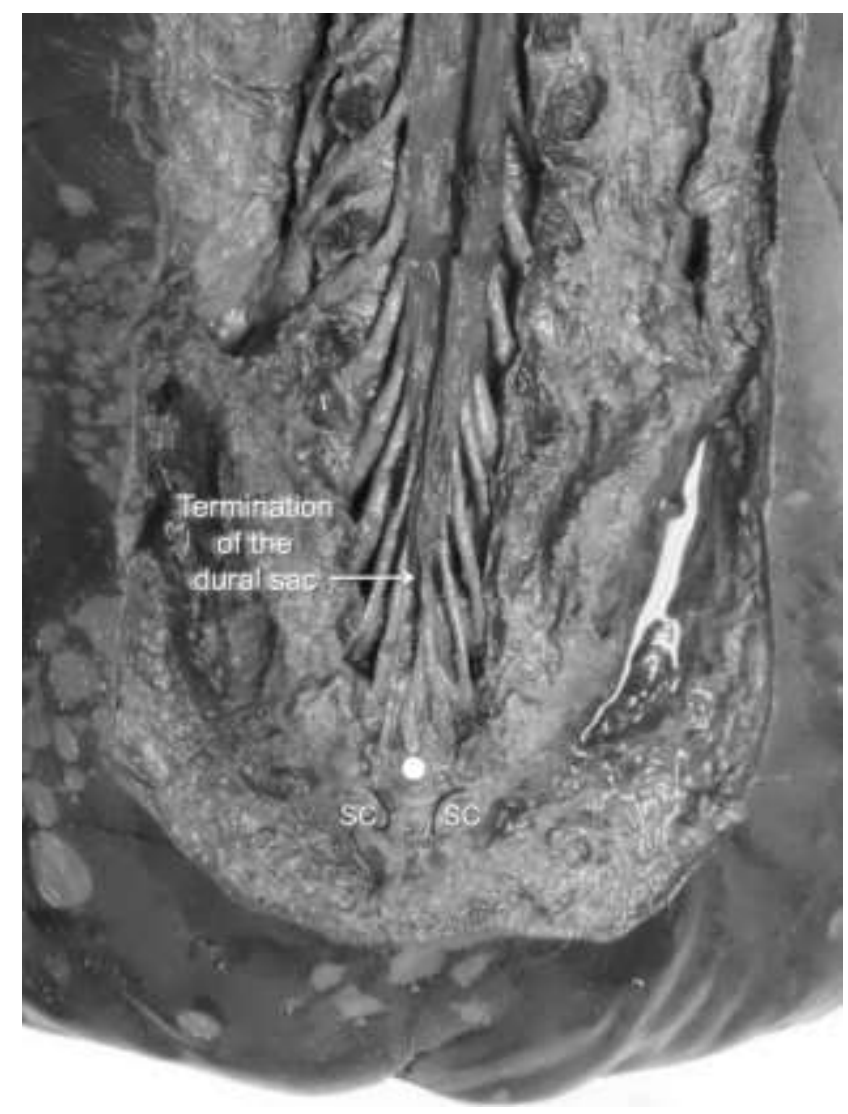

Figure 1: Content of the vertebral canal with the laminae and spinous processes removed in order to show the termination of the dural sac. The sacral cornua (SC) and apex of the sacral hiatus (white dot) are also visible.

A scale of known dimension was placed on top of the dissected area (without covering any of the relevant structures). High quality digital photographs were then taken of the exposed dural sac and surrounding structures.

The photographs were imported into UTHSCSA Image Tool V3.0 to analyze the photographs. Using the Calibrate Spatial Measurements function, the known scale (found on each photograph) was converted into a pixel format by Image Tool. This allowed for accurate measurements of the photographs by means of the Distance function, which converts the length of a straight line, drawn between two points on the photograph, into millimeters. The distance from the apex of the sacral hiatus to the termination of the dural sac was then measured and captured in a Microsoft Excel ${ }^{\mathrm{TM}}$ spreadsheet for statistical analysis. Descriptive statistics were used to describe the data obtained from the cadaveric sample; this included the means and standard deviations of all the measurements. 
The ranges, medians and interquartile ranges were also measured, since the measurements were skewed to the right. The linear relationship between the $\log _{\mathrm{e}}$ of the distance between the apex of the sacral hiatus to the termination of the dural sac (dependent variable) and the length of the cadaver sample in $\mathrm{cm}$ (independent variable) was assessed using linear regression. For the interpretation of the coefficients obtained, these were transformed back using the formula: Percentage change in the distance from the apex of the sacral hiatus to the dural sac $=100\left(e^{b}-1\right)$ where $e$ is the exponential constant.

\section{Results}

The mean distance between the apex of the sacral hiatus and the termination of the dural sac was $10.90 \mathrm{~mm}$ in males $(n=25 ; S D=4.58)$ and $9.64 \mathrm{~mm}$ in females $(n=14 ; S D=2.61)$. The ranges for the readings were 4.94 to 26.28 and 6.66 to 15.09 respectively. There was considerable overlap between the distances for the two sexes, and so the results of the two samples were combined for a total sample of 39. For the total sample the mean distance from the apex of the sacral hiatus to the termination of the dural sac was $10.45 \mathrm{~mm}$ with a standard deviation of $3.99 \mathrm{~mm}$. The range for the combined group was the same as that for the males, namely from 4.94 to 26.2 .

Linear regression was carried out with the $\log _{\mathrm{e}}$ of the distance between the apex of the sacral hiatus and termination of the dural sac regressed on the length in $\mathrm{cm}$, and sex. Weight was not included since weight and length had a Pearson's correlation coefficient of $0.81(p<0.001)$; it seemed more plausible that weight depended on length and not the other way around, and so weight was excluded in favor of length.

The coefficient for sex was not statistically significant $(p=0.333)$ and so sex was excluded from the regression model. The coefficient for the $\log _{e}$ ASHDS and length was $0.033(p<0.001)$. The $R^{2}$ value was 0.39 . This result gives the following percentage change in the measured distance for each $1 \mathrm{~cm}$ increase in length: $3.30 \%$ increase (95\% Confidence Interval: $1.91 \%$ to $4.71 \%)$ for each $1 \mathrm{~cm}$ increase in length. 


\section{Discussion}

Complications are more likely to occur while performing caudal blocks in neonates and infants compared to any other age group (8). Location of the dural sac relative to apex of the sacral hiatus is therefore important since most anesthesiologists change the angle of insertion and advance the needle after penetration of the sacrococcygeal membrane when performing a caudal block. Historically this is a technique derived and modified from adult practice.

Crighton and colleagues suggested that to increase the chances of successful caudal blockade with minimal risk of dural puncture, the most frequent termination level of the dural sac should be known. This is also applicable if the mean distance to the dural sac is known. They stated that by understanding the dimensions of the dural sac, the reliability as well as the safety of caudal procedures is increased (10). Even though their study examined the caudal space in 37 adults by means of MRI, the conclusions are valid and it stands to reason that it is of clinical importance that the distance to the dural sac be known before attempting a caudal block in neonates.

The termination of the dural sac has been the subject of a number of studies using different techniques in the past. The majority is based on early cadaver studies in adults (11), conventional radiographic studies (12), or myelography (13). These studies showed a large variation in the level at which the dural sac terminates and range from $L 5 / S 1$ to $S 4$, with $S 2$ being the most frequent.

Although the exact vertebral level of the termination of the dural sac could not be determined in this study, the results show that the dural sac terminates approximately $10.5 \mathrm{~mm}$ from the apex of the sacral hiatus. This is important information for anesthesiologists performing caudal blocks, particular those who advance the needle into the caudal space after initial penetration of the sacrococcygeal membrane. These results also suggest that the needle should not be advanced more than $4 \mathrm{~mm}$ in neonates to avoid dural puncture. Better still there is no need to change the angle after penetration of the sacrococcygeal membrane - the "no turn technique" - as described by Ivani (14). 
Ultrasound imaging is increasingly being used in modern anesthesia and is a useful modality for performing caudal blocks in young children $(15,16)$ as well as in adults $(17)$. However, calcification of the posterior vertebral bodies in children older than approximately 15 months reduces the acoustic window and makes visualization of the spinal cord and dural sac in older children and adults difficult.

Since the sacrum and vertebrae are not yet fully ossified in neonates and young infants (18) the termination of the dural sac can be easily visualized (19). In this age group, ultrasound can be used to confirm the location of the needle tip or catheter within the caudal space in relation to the surrounding content, i.e., the dural sac and the cauda equina $(16,19)$. The findings in this study are similar to those of Willschke et al. (19) and confirms that the use of ultrasound is reliable when determining the position of the conus medullaris and dura sac in this age group

Lee and co-workers studied the caudal canal and dural sac on MRI scans of 141 pediatric patients (mean age: 20 months) and found that the distance from the apex of the sacral hiatus to the termination of the dural sac was between $12 \mathrm{~mm}$ and $54 \mathrm{~mm}$. This sample was older and larger than our sample. Yet despite this difference, Lee et al. came to the conclusion that even through the dural sac was never found less than $10 \mathrm{~mm}$ from the apex of the sacral hiatus, one should never insert a caudal needle more than $2 \mathrm{~mm}$ to $4 \mathrm{~mm}$ once the saccrococcygeal ligament has been pierced (20). This recommendation is further corroborated by the results of our study where the closest distance between the apex of the sacral hiatus and the dural sac was $4.94 \mathrm{~mm}$ in a male neonate weighing a mere $0.8 \mathrm{~kg}$. It is also important to keep in mind the location of the dural sac changes with the position of the patient and is highest in the flexed knee chest position (21).

Despite the clear benefits of ultrasound guidance, a sound knowledge of the sonar anatomy displayed on the monitor remains crucial in order to perform a successful ultrasound guided block. Unfortunately, ultrasound is not universally available, particularly in low and middle-income countries (22).

There are limitations to this study. Identification of the exact vertebral level of the dural sac in these cadavers was challenging. Embryologically, during stage 23, i.e. 53 - 58 days post fertilization, the 
cartilaginous parts of the sacral vertebrae have fused. The individual pedicles and laminae are very small and might be detected in vertebrae S3 - S5. The ossification of the sacral vertebrae only occurs after week 16 over a time period of 2 to 3 weeks, by which time the second sacral vertebra will be ossified (23). This embryological development of the sacrum resulted in our inability to accurately identify the separate sacral vertebrae in these neonatal cadavers. Furthermore, measurements were made on embalmed neonatal cadavers, which could alter the underlying anatomy and potentially cause shrinkage or anatomical distortions. These distortions are negligible as the anatomy seen in a cadaveric sample is similar to living studies (24). The cadavers used in this study were preterm or fullterm neonates who had died from perinatal complications. Most of the cadavers were of very low or low birth weight neonates with an average weight of $1.61 \mathrm{~kg}$. The anatomy of infants is closer to the anatomy of neonates than to that of an adult sample and we suggest that extrapolating data obtained from neonates is more appropriate for infants and young children than those gathered from adult studies (25).

\section{Conclusion}

Anesthesiologists should be aware of the short distance between the sacral hiatus and the dural sac when performing caudal blocks. Based on the results of this study, the technique of changing the angle and advancing the caudal needle further into the caudal space is brought into question (20). Anesthesiologists who advance the caudal needle more than $5 \mathrm{~mm}$ in neonates are at increased risk of causing an inadvertent dural puncture, particularly if sharp intravenous needles are used.

In the event of a dural puncture, the needle or the caudal catheter must be withdrawn to avoid the risk of a total spinal anesthetic if the full dose were to be injected (9). Options at this point are to convert to spinal anesthetic by injecting a considerably smaller dose without withdrawing the needle or to attempt the caudal block by inserting the needle at a point more caudad.

\section{Acknowledgements}

The authors kindly thank all the body donors without whom this research would not have been possible. We would also like to express our gratitude to their families. 


\section{Disclosures}

Author Adrian Bosenberg is a section editor of Pediatric Anesthesia.

\section{Financial assistance}

The financial assistance of the National Research Foundation (NRF) toward this research is hereby acknowledged. Opinions expressed and conclusions arrived at are those of the authors and are not necessarily attributed to the NRF. Thank you to Prof. Brendan Girdler-Brown, Extraordinary Professor, School of Health Systems and Public Health, University of Pretoria, South Africa for his valued assistance with the statistical interpretation of our data.

\section{Conflict of interests}

None of the authors have any conflicts of interest to declare.

\section{Disclosures}

\section{Ethical approval}

All cadavers were obtained according to the South African National Health Act, number 61 of 2003 and stored in the Department of Anatomy, University of Pretoria, South Africa for research and teaching purposes. Ethical clearance to perform this study (dissection of neonatal cadavers) was obtained from the Research Ethics Committee of the Faculty of Health Sciences, University of Pretoria.

\section{Source of funding}

The financial assistance of the Department of Anatomy, as well as the National Research Foundation (NRF) toward this research is hereby acknowledged. Opinions expressed and conclusions arrived at are those of the authors and are not necessarily attributed to the NRF. 
Conflict of interest

Adrian Bösenberg is a Section Editor for the journal Pediatric Anesthesia. There are no further conflicts of interest to report.

\section{References}

1. Fortuna A. Caudal analgesia: a simple and safe technique in paediatric surgery. $B r J$ Anaesth 1967; 39:165-170.

2. Busoni P, Andreuccetti T. The spread of caudal analgesia in children: a Mathematical model. Anaesth Intens Care 1986; 14:140-144.

3. Adewale L, Dearlove O, Wilson B, Hindle K, Robinson DN. The caudal canal in children: a study using magnetic resonance imaging. Pediatr Anesth 2000; 10:137-141.

4. Dalens B, Hasnaoui A. Caudal anesthesia in pediatric surgery. Anesth Analg 1989; 68:83-89.

5. Broadman LM, Hannalah RS, Norden RS, McGill WA. "Kiddie caudals" experience with 1154 consecutive cases without complications. Anesth Analg 1987; 66:S18.

6. Giaufre E, Dalens B, Gombert A. Epidemiology and morbidity of regional anesthesia in children: a one year prospective survey of the French-language Society of Pediatric Anesthesiologists. Anesth Analg 1996; 83:904-912.

7. Veyckemans F, Van Obbergh LJ, Gouverneur JM. Lessons from 1100 pediatric caudal blocks in a teaching hospital. Reg Anesth 1992; 17(3):119-125.

8. Suresh S, Long J, Birmingham PK, De Oliveira GS Jr. Are caudal blocks for pain control safe in children? an analysis of 18,650 caudal blocks from the Pediatric Regional Anesthesia Network (PRAN) database. Anesth Analg 2015; 120(1):151-156.

9. Dalens BJ. Caudal anesthesia. In: Dalens BJ, editor. Regional Anesthesia in Infants, Children, and Adolescents. Williams \& Wilkens, London, 1995: 171-192.

10. Crighton IM, Barry BP, Hobbs GJ. A study of the anatomy of the caudal space using magnetic resonance imaging. Br J Anaesth 1997; 78:391-395.

11. Hansasuta A, Tubbs RS, Oakes WJ. Filum terminal fusion and dural sac termination: study in 27 cadavers. Pediatr Neurosurg 1999; 30:176-179. 
12. Dunbar SF, Barnes PD, Tarbell NJ. Radiologic determination of the caudal border of the spinal field in cranial spinal irradiation. Int J Radiat Oncol Biol Phys 1993; 26:669-673.

13. Larsen JL, Olsen KO. Radiographic anatomy of the distal dural sac. A myelographic investigation of dimensions and termination. Acta Radiol 1991; 32:214-219.

14. Ivani G. Caudal block: the 'no turn technique'. Pediatr Anesth 2005; 15(1):83-84

15. Yoon JS, Sim KH, Kim SJ, Kim WS, Koh SB, Kim B. The feasibility of color Doppler ultrasonography for caudal epidural steroid injection. Pain 2005; 118:210-214.

16. Siddiqui A. Caudal blockade in children. Tech Reg Anesth Pain Manage 2007; 11:203-207.

17. Blanchais A, Le Goff B, Guillot P, Berthelot J, Glemarec J, Maugars Y. Feasibility and safety of ultrasound-guided caudal epidural glucocorticoid injections. Joint Bone Spine 2010; 77:440-444.

18. Scheuer L, Black S. Developmental Juvenile Osteology. Oxford: Elsevier Academic Press. 2000.

19. Willschke H, Bösenberg A, Marhofer P, Willschke J, Schwindt J, Weintraud M, et al. Epidural catheter placement in the neonates: sonoanatomy and feasibility of ultrasonographic guidance in term and preterm neonates. Reg Anesth Pain Med 2007; 32:34-40.

20. Lee HJ, Min JY, Kim HI, Byon HJ. Measuring the depth of the caudal epidural space to prevent dural sac puncture during caudal block in children. Pediatr Anesth 2017; 27(5):540544.

21. Koo BN, Hong JY, Kim JE, Kil HK. The effect of flexion on the level of termination of the dural sac in paediatric patients. Anaesthesia 2009; 64(10):1072-1076.

22. Möhr D. When will the sonar ping reach our shores? SA J Anaesth Analg 2010; 16:4.

23. Standring S, editor. Gray's Anatomy: the Anatomical Basis of Clinical Practice, 41st edn. New York: Elsevier Limited, 2016.

24. Bösenberg AT. Regional anaesthesia in children: an update. South Afr J Anaesth Analg 2013; 19:282-288.

25. Van Schoor A-N, Bosman MC, Bösenberg AT. Descriptive study of the differences in the level of the conus medullaris in four different age groups. Clin Anat 2015; 25(5):638-644. 